\title{
ESTABILIDADE TEMPORAL DE MEDIDAS DO TEOR E DO POTE NCIAL MÁTRICO DA ÁGUA NO SOLO EM UMA TRANSEÇÃO(1)
}

\author{
J osé Fernandes de Melo Filho(2) \& Paulo Leonel Libardi ${ }^{(3)}$
}

\begin{abstract}
RESUMO
O elevado nível de variabilidade espacial de observações experimentais de campo da condutividade hidráulica do solo dificulta o planejamento de amostragem e a uti lização e extrapolação de valores médi os, em estudos de campo de dinâmica da água no solo. Nesse sentido, o objetivo deste trabal ho foi verificar a estabilidade temporal de medidas do teor e do potencial mátrico da água no solo e sua aplicação como ferramenta para racionalizar a estratégia de amostragem para avaliação da condutividade hidráulica do solo em condições decampo. $O$ experi mento de drenagem foi desenvolvido num Latossolo Vermel hoAmarelo em Piracicaba (SP), numa parcela experimental com 50 pontos de observação distanciados de $1 \mathrm{~m}$ entre si, nos quais foram medidos o teor de água (com TDR) e o potencial mátrico (com tensiômetros) durante três semanas de redistribuição da água no solo. Os resultados mostraram que o método da estabilidade temporal permite identificar, com exatidão, os locais mais adequados para as amostragens, possibilitando a redução do número de amostras necessárias e do custo de execução do esforço amostral para o planejamento de sistemas de uso e manejo de água na agricultura.
\end{abstract}

Termos de indexação: perfil instantâneo, variabilidade espacial, amostragem.

(1) Parte da Tese do primeiro autor, apresentada ao Curso de Pós-Graduação em Solos e Nutrição de Plantas da Escola Superior de Agricultura "Luiz de Queiroz" da Universidade de São Paulo - ESALQ/USP. Pesquisa financiada pela FAPESP. Resumo apresentado na XIV RBMCSA, Campo Grande 2002. Recebido para publicação em novembro de 2003 e aprovado em maio de 2005.

(2) Professor da Escola de Agronomia da Universidade Federal da Bahia - UFBA. CEP 44.380-000 Cruz das AImas (BA). E-mail: jfmelo@ufba.br

(3) Professor da Escola Superior de Agricultura "Luiz de Queiroz" da Universidade de São Paulo - ESALQ/USP. Av. Pádua Dias 11, CEP 13418-900 Piracicaba (SP). Bolsista do CNPq. E-mail: pllibard@esalq.usp.br 


\title{
SUMMARY: TEMPORAL STABILITY OF WATER CONTENT AND WATER MATRIC POTENTIAL IN A FIELD SOIL
}

\begin{abstract}
The high spatial variability of the soil hydraulic conductivity as a function of soil water content (or soil-water matric potential) makes the sampling planning and the use or extrapolation of mean values obtained in field studies of soil-water dynamics difficult. In this context, the objective of this study was to verify the temporal stability of soil-water content and soil -water matric potential and to show how thetemporal stability methodology can be used as a tool to rationalize the sampling strategy of soil hydraulic conductivity under fiedd conditions. A drainage experiment was carried out on an Oxisol, in Piracicaba (SP), Brazil, on an experimental plot with 50 observational points in a straight linespaced 1 meter apart, where soil-water contents and soil-water matric potential were measured with TDR probes and tensiometers, respectively, during threeweeks of water redistribution in the soil. Results showed that the temporal stability method identifies the best sampling locations precisely, making it possible to reduce the number of necessary samples and the costs invested in sampling to establish water useand management systems for agriculture.
\end{abstract}

Index terms: instantaneous profile, spatial variability, sampling.

\section{INTRODUÇÃO}

O sol o é um corpo natural e complexo, resultante da interação dos seus fatores de formação (material de origem, clima, organismos, rel evo e tempo) que o torna um sistema dinâmico e variável, fundamental para a sustentação das diversas formas de vida no planeta.

Dentre as propriedades do solo, seguramente, a condutividade hidráulica está entre aquelas que apresentam maior índice de variabilidade. Resultados de vários autores mostram que a condutividadehidráulica pode apresentar até $190 \%$ de variação para o solo saturado e alcançar até 3.300 \% para a condi ção de não-saturação (Warrick \& Nielsen, 1980; Anderson \& Cassel, 1986).

Estes níveis de variação geram várias conseqüências práticas. Primeiramente, indicam que a utilização e extrapolação de valores médios, resultantes de observações experimentais de campo em estudos de dinâmica da água no sol o, geram erros cuja ordem de grandeza ésignificativamenteel evada (Nielsen et al., 1973; Cadima et al., 1980; J ong van Lier \& Libardi, 1999). Outra conseqüência está relacionada com a determinação do número de amostras necessárias para a estimativa de médias com exati dão adequada. N este caso, o número pode alcançar valores impraticáveis de até 26.000 amostras (Anderson \& Cassel, 1986).

Percebendo esta dificuldade prática para a obtenção de médias representativas de propriedades do sol o com el evado grau de variabilidade, Vachaud et al. (1985) propuseram uma metodologia capaz de reduzir o número de observações requeridas para caracterizar uma propriedade física do solo.
Trabal hando com os dados de teor de água no solo, esses autores introduziram o conceito deestabilidade temporal. Tal conceito, conforme bem explicitaram van Wesenbeeck et al . (1988), pode ser definido como a associação, constante com o tempo, entre a localização espacial e as medidas estatísticas que caracterizam determinada propriedade do sol o. Para o teor de água no solo, este conceito foi comprovado, na medida em que o teor de água em um local mais úmidotendea se manter, assim, em qual quer tempo (Gonçalves et al., 1999).

Kachanoski \& De J ong (1988) aplicaram o conceito deestabilidadetemporal como definido por Vachaud et al. (1985) e concluíram que a armazenagem deágua em determinadolocal resulta da ocorrência de um conjunto de processos hidrológicos que operam em diferentes escalas espaciais, demonstrando, com isso, que análises de coerência espacial poderiam ser usadas para examinar a estabilidade temporal de acordo com a escala espacial de qualquer variável do solo. Suas conclusões significaram um avanço ao introduzir o componente espacial na avaliação da estabilidade temporal definida por Vachaud et al. (1985).

Gonçalves et al. (1999) comprovaram o conceito de Vachaud et al. (1985), quando estudaram a estabilidade temporal da distribuição espacial do teor de água no sol o em uma área irrigada. Em seu trabalho, os autores constataram a persistência das distribuições espaciais do teor de água no solo e a possibilidade de identificar locais de amostragem cujos valores permitam estimar a média geral do teor de água em uma área, em qualquer tempo. Estas conclusões apresentam importantes conseqüências práticas relacionadas com o monitoramento da água e com o uso eficiente de sistemas de irrigação. 
Assim, pode-se afirmar que a verificação da estabilidade temporal pode ser utilizada para responder às crescentes questões sobre problemas relativos à amostragem para medição de propriedades do solo com alta variabilidade, podendo-se alcançar a solução dos problemas contemporâneos, relativos à condutividadehidráulica, com equilíbrio entre o econômico e o técnico, e gerando informações científicas mais claras e precisamente analisadas, cujos resultados poderão ser utilizados com grande confiança.

O objetivo destetrabal hofoi verificar a estabilidade temporal de medidas do teor de água e do potencial mátrico da água no solo em uma transeção, bem como sua aplicação, como ferramenta, para racionalizar a estratégia de amostragem para avaliação da condutividade hidráulica do solo em condições de campo.

\section{MATERIAL E MÉTODOS}

\section{Metodologia de campo}

O experimento foi realizado no campus da Escola Superior de Agricultura Luiz de Queiroz - ESALQ/ USP, localizado no município de Piracicaba (SP), cujas coor denadas geográficas são as seguintes: $22^{\circ}$ $42^{\prime} 30$ " de latitude Sul, $47^{\circ} 38^{\prime} 00^{\prime}$ "de longitude Oeste e altitude de $580 \mathrm{~m}$. O relevo local é plano com declive na área experimental menor que $0,01 \mathrm{~m} \mathrm{~m}^{-1}$. Utilizou-se um Latossolo VermelhoAmarelo álico A moderado textura média (Typic Hapludox). Apresenta-se moderadamente a bem drenado e o uso atual é com pastagem de capimelefante. Algumas características físicas referentes ao solo estudado estão registradas no quadro 1.

A parcela experimental tinha um comprimento de 70 m euma largura de 20 m; na sua linha central, ao longo do comprimento, foram selecionados 50 pontos de observação, distanciados de 1 m entre si e, em cada um deles, instalados cinco tensi ômetros, eqüidistantes, numa circunferência de raio de
$0,10 \mathrm{~m}$, com suas cápsulas porosas local izadas nas profundidades de 0,$15 ; 0,30 ; 0,45 ; 0,60 ; 0,75 \mathrm{~m}$ (Figura 1). No centro da circunferência de cada conjunto dos cinco tensiômetros, foi instalada uma sonda de "time domain reflectrometry" (TDR) para medida do teor de água no solo (Toop et al., 1980; Thomsen et al., 2000) nas mesmas cinco profundidades. Manômetros demercúrio, conectados aos tensiômetros, bem como os correspondentes cabos de TDR, foram localizados fora da área útil experimental. Antes da instalação dos equipamentos, a vegetação de capim-el efante foi roçada equeimada, assim como foram realizadas uma aração e uma gradagem na camada de $0-0,30$ m para uniformizar a área experimental.

Os tensiômetros utilizados foram construídos com tubos dePVC rígi do com diâmetro externo e interno, medindo 21 e $16 \mathrm{~mm}$, respectivamente, ecomprimento correspondenteà profundidade deinstalação. U ma tubulação de plástico ("nylon"), com diâmetro interno de $2 \mathrm{~mm}$, também colada ao tubo de PVC, foi

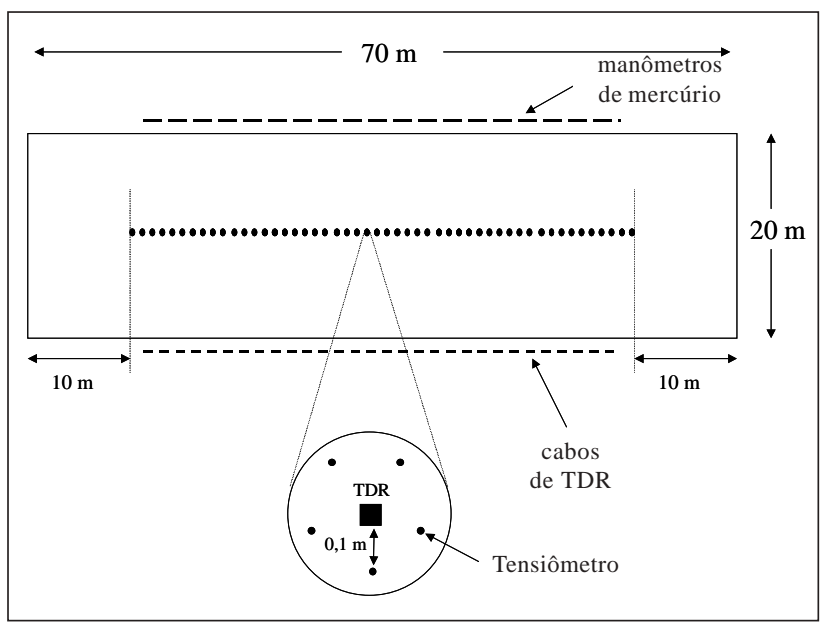

Figura 1. Parcela experimental com a localização dos 50 pontos de observação. 0 detal he mostra a configuração de um ponto de observação com TDR e tensiômetros.

\section{Quadro 1. Textura e granulometria dos horizontes descritos no perfil de solo da área experimental}

\begin{tabular}{|c|c|c|c|c|c|c|}
\hline \multirow{2}{*}{ Horizonte (Prof.) } & \multirow{2}{*}{ Argila } & \multirow{2}{*}{ Silte } & \multicolumn{3}{|c|}{ Areia } & \multirow{2}{*}{ Classificação } \\
\hline & & & Grossa & Fina & Total & \\
\hline & & & $\%$ & 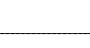 & 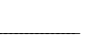 & \\
\hline Ap $(0-0,15 \mathrm{~m})$ & 16,7 & 8,4 & 12,9 & 62,0 & 74,9 & Franco-arenoso \\
\hline$A B(0,15-0,31 \mathrm{~m})$ & 18,6 & 6,2 & 14,8 & 60,4 & 75,2 & Franco-arenoso \\
\hline BA $(0,31-0,50 \mathrm{~m})$ & 18,8 & 6,3 & 15,6 & 59,3 & 74,9 & Franco-arenoso \\
\hline B $1(0,50-0,78 \mathrm{~m})$ & 23,1 & 10,5 & 14,7 & 51,7 & 66,4 & Franco-arenoso \\
\hline B2 $(0,78-1,08 \mathrm{~m})$ & 27,9 & 4,3 & 13,9 & 53,9 & 67,8 & Franco-argilo arenoso \\
\hline B $3(+1,08 \mathrm{~m})$ & 25,4 & 6,4 & 13,6 & 54,6 & 68,2 & Franco-argilo-arenoso \\
\hline
\end{tabular}


utilizada para conectar cada um dos cinco tensiômetros de cada ponto a uma única cuba com mercúrio dos cinco manômetros deleitura do ponto.

As sondas de TDR utilizadas foram da empresa E.S.I. Environmental Sensors I nc., "Type" 4 (PRB-H), model MP 917, com cinco segmentos de 0,15 m para medida do teor deágua. A partir deum conjunto de dados de 210 pares de leituras de TDR $\times \theta$ (teor de água no solo), foram ajustadas equações de cal ibração para cada profundidade e cada ponto de observação.

Para o ensaio do perfil instantâneo, o solo da parcela experimental foi umedecido, aplicando-seágua por meio de irrigação por aspersão, continuamente, durante cinco dias, até os tensiômetros indicarem leitura constante. Em seguida, a superfície do solo foi coberta com uma Iona plástica, satisfazendo, assim, a primeira condição de contorno exigida pelo método do perfil instantâneo. A partir desse momento eduranteo processo der edistribuição, o teor de água no solo $(\theta)$ e o potencial mátrico da água no solo $\left(\phi_{m}\right)$ foram monitorados pelas leituras dos TDRs e dos tensiômetros (Libardi et al., 1980; Libardi, 2000). O período considerado para as análises e avaliações deste estudo ficou compreendido entre os tempos de 78 e 604 h de redistribuição da água, após a cobertura da superfície do sol o com a lona plástica $(t=0)$, correspondente à parte dos meses de junho e julho de 2000. Neste período, foram realizadas 12 medições de $\theta$ e $\phi_{m}$. Em decorrência de defeitos nas sondas de TDR e, ou, tensiômetros, cinco pontos de observação foram descartados para as análises deste trabalho.

\section{Estabilidade temporal: conceitos e teoria}

Para aplicar o conceito de estabilidade temporal a um conjunto de dados, é necessário que duas condições sejam atendidas. A primeira condição requer, para determinado tempo, um número suficiente de observações para obtenção de seus parâmetros estatísticos clássicos. Na segunda condição, deve ser possível associar a cada posição ou local o seu valor estatístico de densidade de probabilidade de distribuição normal. Existindo estas duas condições, a estabilidade temporal de um conjunto de dados pode ser analisada, aplicando-se dois procedimentos (Vachaud et al. 1985). O primeiro, denominado cálculo da diferença relativa, faz uma análise dos desvios entre os valores observados individualmente no espaço e a média deles. I gualdades ou pequenas variações da diferença relativa entre posições ao longo do tempo indicam a estabilidade temporal. A segunda técnica é o teste de correlação de Spearman (Campos, 1983) que é utilizado como uma ferramenta estatística para indicar o grau de concordância da variabilidade espacial obtida em diferentes tempos.

O teste não-paramétrico de Spearman é um procedimento eficiente que possibilita calcular o grau de dependência entre duas variáveis al eatórias. Um val or der igual à unidade corresponderá à igualdade de posição para qualquer local ou estabilidade perfeita entre duas datas ou tempos. Quanto mais próximo de 1 for o $r$, mais estável será o processo (Campos, 1983; Vachaud et al., 1985).

O coeficiente de correlação de Sperman ( $r$ ) possibilita verificar a existência da estabilidade temporal, mas não identifica os locais em que as medi das possam ser feitas para representar a média da variável em estudo para qual quer tempo e valor. Para tanto, Vachaud et al. (1985) indicam o cál culo das diferenças relativas (DR) e seus respectivos desvios-padrão. As diferenças relativas, quando ordenadas e plotadas em um gráfico, possibilitam identificar os pontos cujos valores sempre estejam próximos da média e possam ser utilizados como referência amostral.

Assim, com os valores do teor deágua no solo $\theta \mathrm{e}$ do potencial mátrico da água no solo $\phi_{m}$, estimados para cada tempo, procedeu-se ao cálculo das diferenças relativas (Vachaud et al., 1985), conforme as seguintes equações: equação (1), para o $\theta$, e equação (2), para o $\phi_{m}$.

$$
\begin{gathered}
\operatorname{DR}(\theta)=\frac{\theta_{\mathrm{ij}}-\bar{\theta}}{\bar{\theta}} \times 100 \\
\operatorname{DR}\left(\phi_{\mathrm{m}}\right)=\frac{\phi_{\mathrm{m}(\mathrm{ij})}-\overline{\phi_{\mathrm{m}}}}{\overline{\phi_{\mathrm{m}}}} \times 100
\end{gathered}
$$

sendo: DR a diferença relativa entre determinação individual para um local e tempo e sua estimativa da média; $\theta$ o teor da água no sol o; $\theta_{\mathrm{ij}}$ o teor de água no local i e no tempo j; $\bar{\theta}$ o teor médio de água para todas as posições, no momento j; $\phi_{m(i j)}$ o potencial mátrico da água no solo no local i no tempo j; $\overline{\phi_{\mathrm{m}}} \mathrm{o}$ potencial mátrico médio da água no sol o para todas as posições, no momentoj. Com objetivo de indicar o grau de confiabilidade da medida também foram determinados os respectivos desvios-padrão relacionados com as variações espaciais.

Depois de cal culadas e ordenadas, as diferenças relativas e seus respectivos desvios-padrão foram plotados em um gráfico, cuja observação permitiu identificar os locais que, sistematicamente, superestimam (DR $>0$ ) ou subestimam $(D R<0)$ as medidas médias da variável em estudo, independentemente do tempo de observação. O local a ser escol hido para a col eta de amostras, cujos valores sejam confiáveis e representativos, deve apresentar uma diferença relativa média igual a zero, ou muito próxima de zero, e estar associada ao menor desvio-padrão (Vachaud et al., 1985; Gonçalves et al., 1999).

Normalmente, os autores que aplicavam essa técnica indicavam apenas uma posição no espaço com estabilidade temporal. No entanto, por se considerar a identificação de mais de uma posição 
para as futuras medições mais interessante, na medida em que introduz um el emento de avaliação estatística, qual seja, a repetição, mesmo que traga consigo algum nível de variação e, para evitar um julgamento subjetivo, compararam-segraficamente os val ores de $\theta$ e $\phi_{m}$, para cada local de amostragem selecionado no gráfico das diferenças relativas, com as médias gerais de cada propriedade, em todo o período de amostragem, estabel ecendo-se que os pontos mais indicados para amostragem e determi nação de $\theta$ e $\phi_{m}$, nas três profundidades, 0,30; 0,45 e 0,60 m seriam aqueles cujo comportamento estivesse mais próximo da média.

Para tanto, seguindo o procedimento sugerido por Vachaud et al. (1985), foram selecionados doze tempos de redistribuição, correspondentes ao período de avaliação experimental entre 78 e 604 h no experimento de perfil instantâneo. I nicialmente, verificou-sea normal idade da distribuição dos dados, calcularam-se os coeficientes de correlação de Spearman (SAS, 1991) para os val ores de $\theta$ e $\phi_{m}$, assim como as diferenças relativas (DR) e seus respectivos desvios-padrão, queforam, posteriormente, plotados em um gráfico em função da posi ção para a identificação dos locais que apresentam estabilidade temporal para $\theta$ e $\phi_{\mathrm{m}}$.

\section{RESULTADOS E DISCUSSÃO}

\section{Estatística exploratória}

Os parâmetros estatísticos para um conjunto de dados de $\theta$ e $\phi_{m}$ sãomostrados no quadro 2 . Deacordo com os resultados da análise estatística expl oratória para $\theta\left(\mathrm{m}^{3} \mathrm{~m}^{-3}\right)$, verificou-se queos val ores estiveram muito próximos durante o período de avaliação nas três profundidades, indicando que, tanto a saturação inicial do solo quanto a secagem durante o processo de redistribuição, foram bem uniformes. Esta homogeneidade das condições experimentais é confirmada pelos baixos coeficientes de variação apresentados por $\theta$ nas três profundidades. Verificou-setambém que o $\theta$ seguiu uma distribuição normal para os quatro tempos de avaliação nas três profundidades.

Por outro lado, os dados de $\phi_{\mathrm{m}}(\mathrm{kPa})$ apresentaram um comportamento bem diferente de $\theta$. Neste caso, houve um aumento da variabilidade, medida pelo desvio-padrão, com o tempo e a normalidade da distribuiçãofoi fortemente afetada pela presença de "outliers". Para confirmar este efeito, aplicou-se o critério definido por Libardi et al. (1996) para identificação dos "outliers", constatando-se que a eliminação dos valores extremos melhorou a distribuição dos dados de $\phi_{m}$, tornando-os normais. Assim, pode-se afirmar que os dados apresentaram distribuição normal e, portanto, enquadram-se nas condições definidas por Vachaud et al. (1985), para que um conjunto de dados experimentais possa ter a sua estabilidade temporal avaliada.

Outro aspecto importante e que deve ser ressaltado refere-se à utilização do coeficiente de variação como parâmetro descritivo da variabilidade do $\phi_{\mathrm{m}}$. Diferentemente do desvio-padrão, o coeficiente de variação indica uma redução da variabilidade de $\phi_{\mathrm{m}}$ com o tempo nas três profundidades (Quadro 2). Certamente, este é um comportamento não relacionado com o fenômeno e

Quadro 2. Resumo estatístico para $\theta\left(\mathrm{m}^{3} \mathrm{~m}^{-3}\right)$ e $\phi_{\mathrm{m}}(\mathrm{kPa})$, em três profundidades e quatro tempos de amostragem, segundo uma transeção de 50 pontos espaçados de um metro

\begin{tabular}{|c|c|c|c|c|c|c|c|c|c|c|c|c|}
\hline \multirow{3}{*}{ Tempo } & \multicolumn{12}{|c|}{ Profundidade } \\
\hline & \multicolumn{4}{|c|}{$0,30 \mathrm{~m}$} & \multicolumn{4}{|c|}{$0,45 \mathrm{~m}$} & \multicolumn{4}{|c|}{$0,60 \mathrm{~m}$} \\
\hline & Média & DP & CV & Distr. & Média & DP & CV & Distr. & Média & DP & CV & Distr. \\
\hline \multirow[t]{2}{*}{$\mathrm{h}$} & & & $\%$ & & & & $\%$ & & & & $\%$ & \\
\hline & \multicolumn{12}{|c|}{ Teor de água no solo $(\theta)$} \\
\hline $\begin{array}{r}78 \\
220 \\
460 \\
604\end{array}$ & $\begin{array}{l}0,25 \\
0,23 \\
0,22 \\
0,21\end{array}$ & $\begin{array}{l}0,011 \\
0,012 \\
0,012 \\
0,010\end{array}$ & $\begin{array}{l}4,44 \\
5,22 \\
5,45 \\
4,76\end{array}$ & $\begin{array}{l}N \\
N \\
N \\
N\end{array}$ & $\begin{array}{l}0,24 \\
0,23 \\
0,21 \\
0,21\end{array}$ & $\begin{array}{l}0,009 \\
0,010 \\
0,009 \\
0,010\end{array}$ & $\begin{array}{l}3,75 \\
4,35 \\
4,29 \\
4,76\end{array}$ & $\begin{array}{l}\mathrm{N} \\
\mathrm{N} \\
\mathrm{N} \\
\mathrm{N}\end{array}$ & $\begin{array}{l}0,24 \\
0,23 \\
0,22 \\
0,21\end{array}$ & $\begin{array}{l}0,014 \\
0,014 \\
0,013 \\
0,013\end{array}$ & $\begin{array}{l}5,83 \\
6,09 \\
5,91 \\
6,19\end{array}$ & $\begin{array}{l}N \\
N \\
N \\
N\end{array}$ \\
\hline 604 & \multicolumn{12}{|c|}{ Potencial mátrico da água no solo $\left(\phi_{\mathrm{m}}\right)$} \\
\hline $\begin{array}{r}78 \\
220 \\
460 \\
604\end{array}$ & $\begin{array}{l}-1,25 \\
-2,39 \\
-3,75 \\
-4,35\end{array}$ & $\begin{array}{l}0,46 \\
0,43 \\
0,42 \\
0,48\end{array}$ & $\begin{array}{l}36,80 \\
17,99 \\
11,20 \\
11,03\end{array}$ & $\begin{array}{l}\mathrm{N} \\
\mathrm{N} \\
\mathrm{N} * \\
\mathrm{~N}^{*}\end{array}$ & $\begin{array}{l}-0,49 \\
-1,72 \\
-3,19 \\
-3,67\end{array}$ & $\begin{array}{l}0,39 \\
0,52 \\
0,72 \\
0,71\end{array}$ & $\begin{array}{l}79,59 \\
30,23 \\
22,57 \\
19,35\end{array}$ & $\begin{array}{l}\mathrm{N} \\
\mathrm{N}^{*} \\
\mathrm{~N}^{*} \\
\mathrm{~N}^{*}\end{array}$ & $\begin{array}{l}-0,48 \\
-1,24 \\
-2,51 \\
-3,08\end{array}$ & $\begin{array}{l}0,44 \\
0,64 \\
0,72 \\
0,71\end{array}$ & $\begin{array}{l}91,67 \\
51,61 \\
28,69 \\
23,05\end{array}$ & $\begin{array}{l}\mathrm{N}^{*} \\
\mathrm{~N} \\
\mathrm{~N} \\
\mathrm{~N}\end{array}$ \\
\hline
\end{tabular}

$\mathrm{DP}$ = desvio-padrão; CV = coeficiente de variação (\%); N = distribuição normal

* Normalidade verificada após a retirada dos "outliers". 
foi decorrente do aumento, em módulo, dos valores depotencial mátrico, oqueénormal eexperimentalmente correto. Desta forma, a relação matemática entre estes índices resultou na redução dos valores do coeficiente de variação. Neste caso, o desviopadrãoéo parâmetro estatístico que melhor descreve o comportamento do $\phi_{m}$, enquanto o coeficiente de variação deve ser utilizado para caracterizá-lo de forma geral, apenas classificando-o quanto ao seu grau de variabilidade. Assim, de acordo com os critérios de Warrick \& Nielsen (1980), verificou-se que os valores do coeficiente de variação do potencial mátrico atingiram todos os níveis de variação (baixo, médio ealto). No início do experimento, o coeficiente de variação foi médio, na profundidade de 0,30 m, e alto, nas profundidades de 0,45 e 0,60 m. Com o tempo, evoluiu para médio e manteve-se assim nas três profundidades até o tempo final da análise (Quadro 2).

As figuras 2 e 3 mostram a heterogeneidade espacial, ao longo da transeção, dos valores de $\theta$ e $\phi_{m}$, respectivamente, nas profundidades de 0,30, 0,45 e $0,60 \mathrm{~m}$. As ilustrações mostram quatro tempos, com destaque para o tempo inicial e final. Detalhando a análise das figuras 2 e 3 , percebeu-se que, tanto para $\theta$ quanto para $\phi_{m}$, os padrões de variabilidade das medidas foram diferentes nas três profundidades. Não obstante, quando se comparou individualmente cada profundidade, verificou-se,

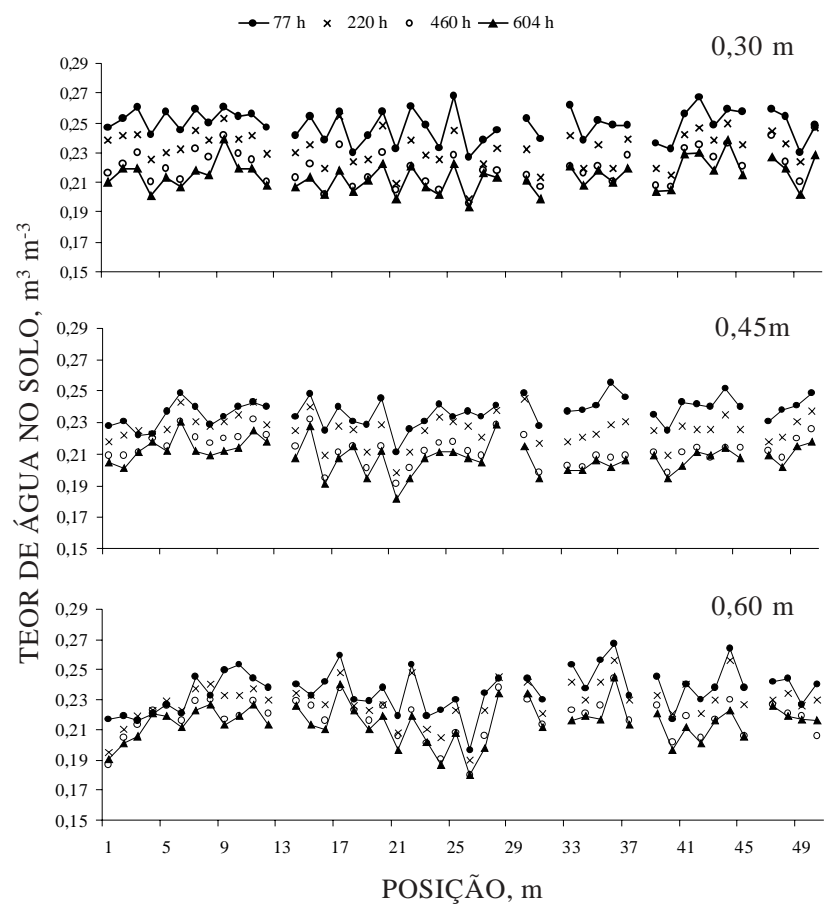

Figura 2. Variabilidade do teor de água no solo $\left(\mathrm{m}^{3} \mathrm{~m}^{-3}\right)$, segundo uma transeção de 50 pontos espaçados de um metro, em três profundidades e quatro tempos de amostragem. A linha interrompida indica os cinco pontos de medida que foram eliminados.

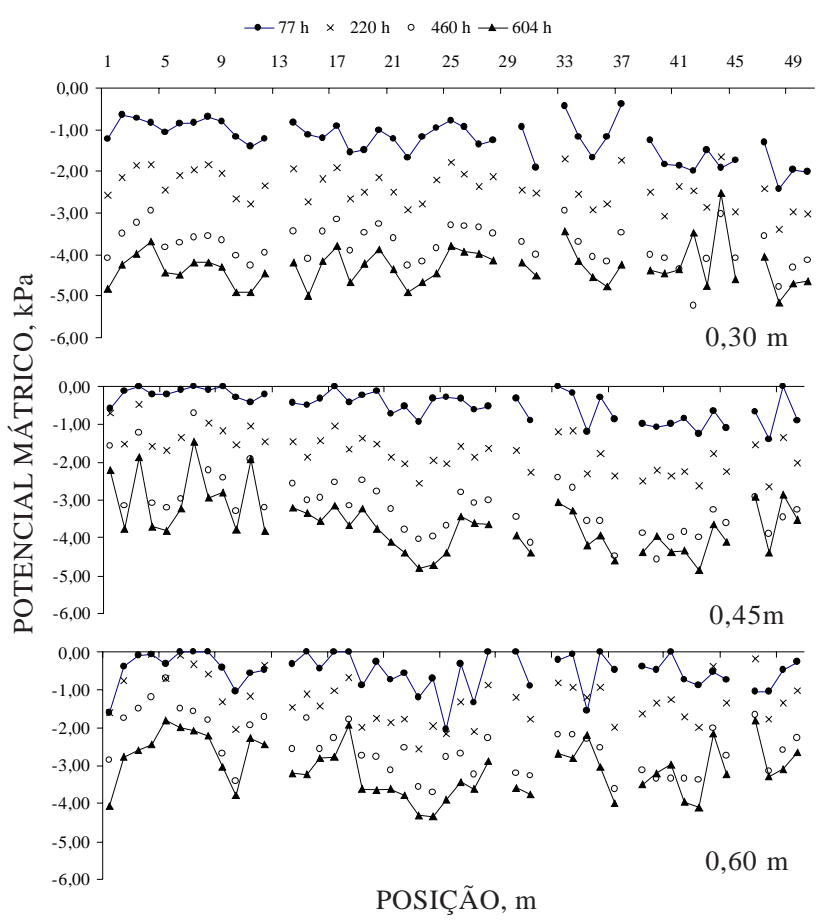

Figura 3. Variabilidade do potencial mátrico (kPa), segundo uma transeção de 50 pontos espaçados de um metro, em três profundidades e quatro tempos de amostragem. A linha interrompida indica os cinco pontos de medida que foram eliminados.

para ambos os casos, uma similaridade no comportamento das medidas durante o processo de redistribuição. Ficou bem claro que a maioria dos pontos manteve semprea mesma posição em relação ao conjunto de medidas. Este comportamento foi mais definido para o $\theta$ do que para o $\phi_{m}$. A maior uniformidade na distribuição das medidas de $\theta$ em relação ao $\phi_{m}$ resultou dos coeficientes de variação apresentados por essas medidas. Para $\theta$, os coeficientes de variação foram mais baixos e semel hantes durantea redistribuição. J á para o $\phi_{m}$, os val ores dos coeficientes de variação foram mai ores e bem diferentes ao longo do tempo. Daí, a diferença de comportamento.

As mesmas ilustrações (Figuras 2 e 3 ) também mostram que as medidas de $\theta$ e $\phi_{m}$ distribuíram-se ao longo da transeção com uma estacionaridade que revela a ausência de qualquer tendência para concentração de valores em determinada direção. Tal fato permitiu assumir que a condição definida pela hipótese intrínseca também pode ser aplicada a este conjunto de dados.

\section{Estabilidade temporal}

Coeficiente de correlação: $O$ quadro 3 mostra os coeficientes de correlação de Spearman $(r)$ para $\theta$ e $\phi_{m}$. De maneira geral, tanto para $\theta$ quanto para $\phi_{m}$, os índices de correlação diminuíram nas três 
profundidades, indicando que a correlação para al guns locais muda significativamente com otempo. Embora alguns valores de $r$ tenham sido baixos, todos os resultados apresentaram correlação significativa a 0,01 de significância (SAS, 1991), indicando a existência de estabilidade temporal forte nas posições com r próximo a 1. De outra forma, aplicando os limites unilaterais de r para o teste de correlação de Spearman (Campos, 1983), a interpretação seria mais sel etiva, tendo em vista o limite crítico de 0,671 para significância de 0,01. Então, obter-se-ia uma redução significativa entre as correlações temporais, principalmente para as medidas de $\phi_{\mathrm{m}}$. Neste caso, para o teor de água no solo, na profundidade de $0,30 \mathrm{~m}$, todos os tempos apresentaram uma correlação significativa; na profundidade de $0,45 \mathrm{~m}$, a correlação existiria até o tempo $268 \mathrm{~h}$ e, na profundidade de $0,60 \mathrm{~m}$, até $460 \mathrm{~h}$. Para o $\phi_{m}$, a correlação não se apresentou linear. Alguns tempos intermediários apresentaram correlação significativa, enquanto outros não. Tal fato pode resultar de diferenças nas relações determinísticas entre essas propriedades e as características do solo, como a textura ea estrutura, cuja comprovação poderá ser feita por meio de análises e avaliações posteriores e que não foram previstas para este estudo.

Diferenças relativas: Um gráfico dos resultados da DR vs "rank" possi bilita a identificação visual dos locais de amostragem que apresentam os valores mais próximos da média em qualquer tempo. O desvio-padrão relacionado com a DR estima o grau de confiabilidade da medida da diferença relativa. Quanto menor o desvio-padrão, mais confiável o valor da DR.
Para o $\theta$ (Figura 4), os locais que apresentaram as menores diferenças relativas e, conseqüentemente, mais se aproximaram do valor médio na transeção foram os seguintes: o ponto 14, na profundidade de $0,30 \mathrm{~m} ; 8,16,37$, na profundidade de $0,45 \mathrm{~m}$, e 34 , na profundidade de 0,60 m. Para o $\phi_{m}$ (Figura 5), os locais que mais seaproximaram da média geral nas três profundidades foram os pontos 5,20 e 31 , na profundidade de $0,30 \mathrm{~m} ; 14,26 \mathrm{e} 28$, na profundidade de $0,45 \mathrm{~m}$ e, finalmente, os pontos 9 e 44, na profundidade de $0,60 \mathrm{~m}$. Para ambos os casos, notouse que não houve coincidência de posições representativas para a estimativa das médias. Este é um aspecto importante para o planejamento da amostragem, tendo em vista que revela uma dependência entre a estabilidade temporal e a profundidade da amostragem, resultando em maior número de posições para execução de um plano de monitoramento da água no solo.

A comparação entre a média geral de $\theta$ e $\phi_{m}$ para todas as 45 posições com os valores dos locais identificados, com base na DR, como estáveis no tempo encontram-se nas figuras 6 e 7, respectivamente. Para os dois casos, pode-se observar que as posições com estabilidade temporal apresentam valores de $\theta$ e $\phi_{m}$ muito próximos da média geral, indicando que o procedimento proposto por Vachaud et al. (1985) realmente é válido e tem exatidão aceitável para utilização como método de amostragem. No entanto, nota-se que as medidas mostram comportamento bem diverso. $\mathrm{N}$ as condições deste estudo, as posições sel ecionadas para $\phi_{\mathrm{m}}$ apresentaram maior exatidão, representada pelo menor afastamento da média geral, especialmente na profundidade de $0,30 \mathrm{~m}$, na qual os valores foram

Quadro 3. Coeficientes de correlação de Spearman ( $r$ ) para diversos tempos de redistribui ção durante o experimento de perfil instantâneo para $\theta\left(\mathrm{m}^{3} \mathrm{~m}^{-3}\right)$ e $\phi_{\mathrm{m}}(\mathrm{kPa})$, em três profundidades, em uma transeção de 50 pontos espaçados de um metro

\begin{tabular}{|c|c|c|c|c|c|c|}
\hline \multirow{2}{*}{ Tempo } & \multicolumn{3}{|c|}{ Teor de água $(\theta)$} & \multicolumn{3}{|c|}{ Potencial mátrico de água $\left(\phi_{\mathrm{m}}\right)$} \\
\hline & $0,30 \mathrm{~m}$ & $0,45 m$ & $0,60 \mathrm{~m}$ & $0,30 \mathrm{~m}$ & $0,45 \mathrm{~m}$ & $0,60 \mathrm{~m}$ \\
\hline $\mathrm{h}$ & 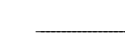 & & 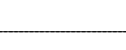 & 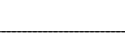 & 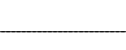 & - \\
\hline 78 & 1,00 & 1,00 & 1,00 & 1,00 & 1,00 & 1,00 \\
\hline 123 & 0,92 & 0,92 & 0,87 & 0,85 & 0,75 & 0,76 \\
\hline 167 & 0,87 & 0,85 & 0,84 & 0,68 & 0,69 & 0,70 \\
\hline 220 & 0,84 & 0,78 & 0,90 & 0,71 & 0,83 & 0,65 \\
\hline 268 & 0,73 & 0,68 & 0,79 & 0,72 & 0,65 & 0,73 \\
\hline 315 & 0,87 & 0,56 & 0,86 & 0,53 & 0,71 & 0,55 \\
\hline 361 & 0,82 & 0,60 & 0,73 & 0,61 & 0,67 & 0,64 \\
\hline 412 & 0,83 & 0,51 & 0,70 & 0,44 & 0,66 & 0,50 \\
\hline 460 & 0,81 & 0,49 & 0,72 & 0,63 & 0,67 & 0,50 \\
\hline 506 & 0,82 & 0,49 & 0,67 & 0,45 & 0,59 & 0,49 \\
\hline 553 & 0,78 & 0,50 & 0,56 & 0,48 & 0,53 & 0,56 \\
\hline 604 & 0,85 & 0,49 & 0,69 & 0,39 & 0,63 & 0,51 \\
\hline
\end{tabular}



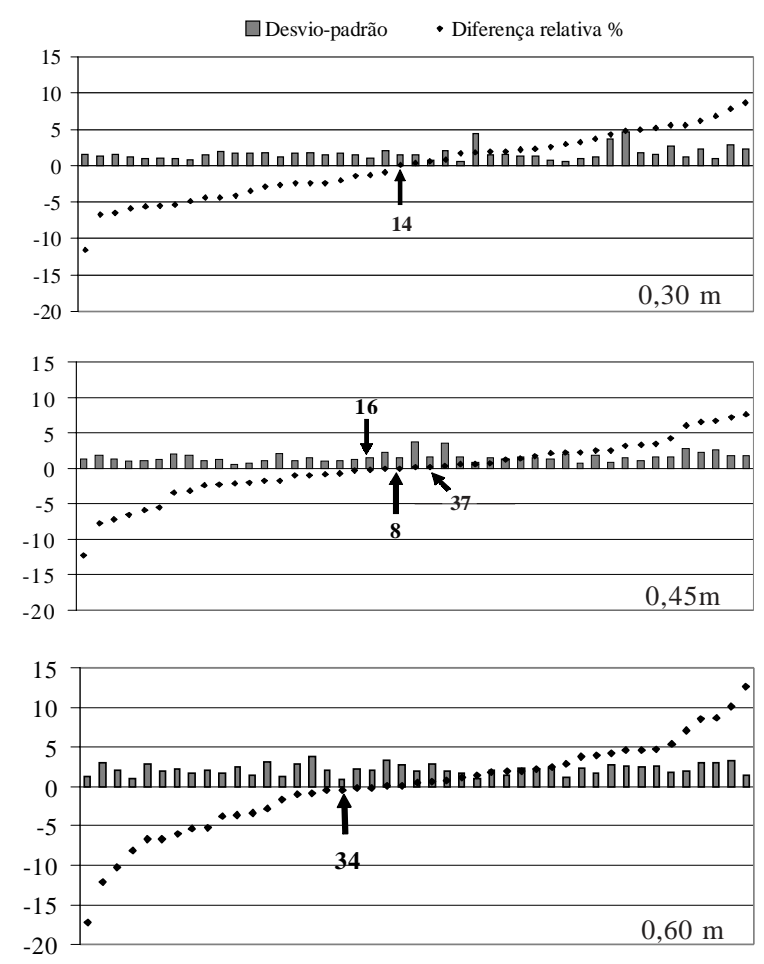

POSIÇÃO, m

Figura 4. Diferença relativa média intertemporal e desvio-padrão para o teor de água no solo $\left(\mathrm{m} \mathrm{m}^{-3}\right)$, em três profundidades, segundo uma transeção de 50 pontos espaçados de um metro. Valores em destaque indicam os pontos que devem ser adotados para medidas representativas da média de umidade no solo.

praticamente os mesmos (Figura 7). Para $\theta$, verificouse que as posições também reproduziam a média geral, porém com menor grau de exatidão. Neste caso, percebeu-se uma leve indicação de que o grau de exatidão das medidas também pode variar com o tempo. A figura 6 revela que as curvas representativas dos pontos estáveis ficam mais afastadas da média geral do experimento em dois momentos. No início da avaliação, quando o solo estava mais úmido e, no final, quando o solo estava mais seco. Esta variação pode estar relacionada com o equi pamento de medida (TDR), mas, certamente, também reflete a contribuição de outros fatores, como a textura e a porosidade do solo, que, seguramente, influem na variabilidade espacial do $\theta$ em uma área. Tambémé possível quea variabilidade torne-se mais evidente com o passar do tempo, conforme verificaram Hendrickx \& Wierenga (1990).

Outro aspecto interessante para aplicação da técnica de Vachaud et al. (1985) que surge desta discussão e que pode mel horar muito a escol ha das posições com estabilidade temporal refere-se à comparação gráfica (Figuras 6 e 7) entre os valores da média geral e os val ores das posições identificadas como estáveis. Esta ação possibilita identificar as posições mais estáveis, posto que pode ocorrer de uma posição apresentar DR próximo de zero, mas não reproduzir a mesma tendência da média, conforme foi verificado para $\theta$ na posição 5 da profundidade $0,30 \mathrm{~m}$ deste experimento.

Não obstante, a aplicação da técnica da estabilidadetemporal para métodos de amostragem apresenta um aspecto prático bem interessante. Demonstra ser possível realizar um processo de amostragem com exatidão e reduzido esforço amostral, mesmo para propriedades com el evado coeficiente de variação como $\phi_{m}$ cujo CV alcançou valores próximos a $90 \%$. Certamente, a utilização de métodos mais comumente empregados (Santos \& Vasconcel os, 1987) resultaria em grande número deamostras, auja exeqüibilidade de obtenção exigiria complexa logística e disponibilidade de recursos financeiros ou a opção por el evado grau de variação em torno da média para redução do número mínimo de amostras requeridas.

Os resultados também permitem afirmar que, para ambos os casos, nestesoloelocalização, qualquer um dos pontos identificados como estáveis no tempo poderia ser escolhido para o monitoramento e col eta

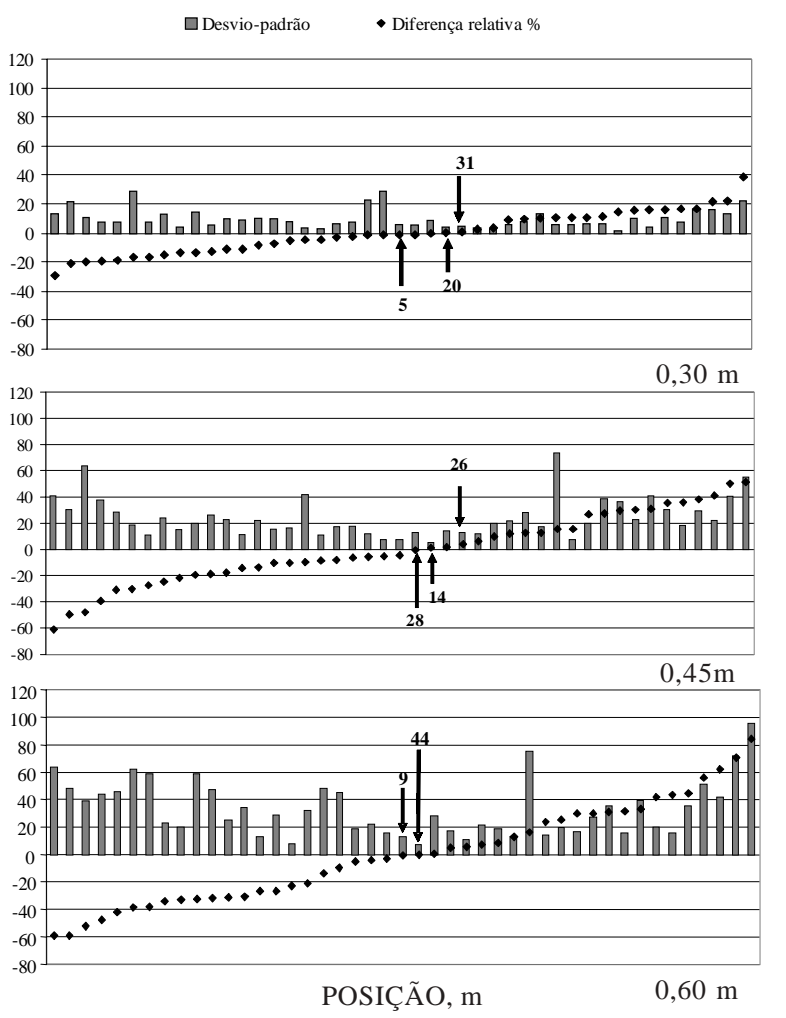

Figura 5. Diferença relativa média intertemporal e desvio-padrão para o potencial mátrico da água no solo ( $\mathrm{kPa}$ ), em três profundidades, segundo uma transeção de $\mathbf{5 0}$ pontos espaçados de um metro. Valores em destaque indicam os pontos que devem ser adotados para medidas representativas da média de potencial mátrico do solo. 


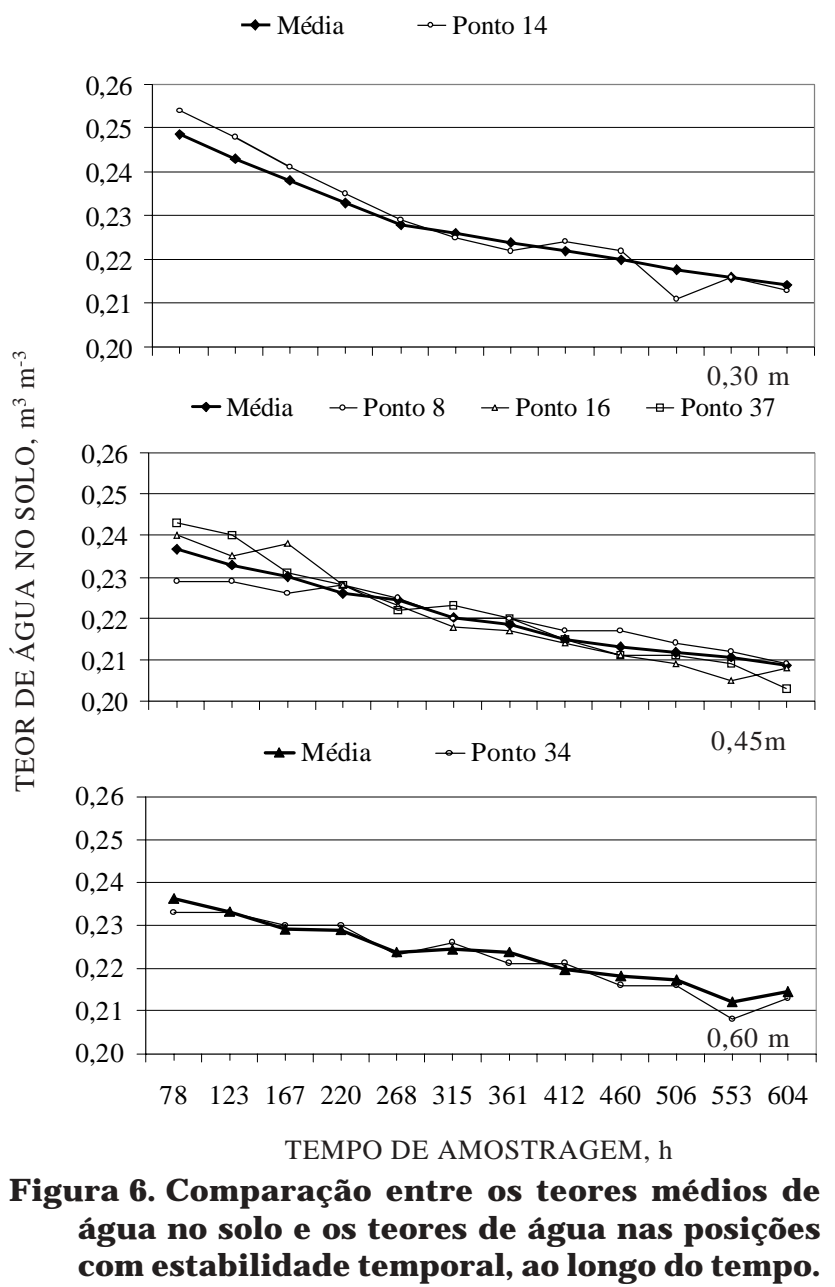

de amostras médias representativas das variáveis $\theta$ e $\phi_{m}$. A existência de mais de um ponto com esta característica, bem como as diferenças entre as profundidades, deve ser atribuída à variabilidade espacial da textura do solo, cuja influência nas medidas de teor de água e potencial mátrico da água no solo é bastante significativa (Vachaud et al., 1985).

\section{CONCLUSÕES}

1. A existência da estabilidade temporal para teor e potencial mátrico da água no solo, durante o tempo de redistribuição da água, no método do perfil instantâneo para determinação da condutividade hidráulica, possibilitou identificar, no campo, posições que representavam as médias dessas variáveis em qualquer tempo. Isso representa um avanço em relação aos outros métodos tradicionais, na medida em que possibilita a redução do número de amostras necessárias para estimar uma média representativa com elevada exatidão e reduzido esforço amostral.

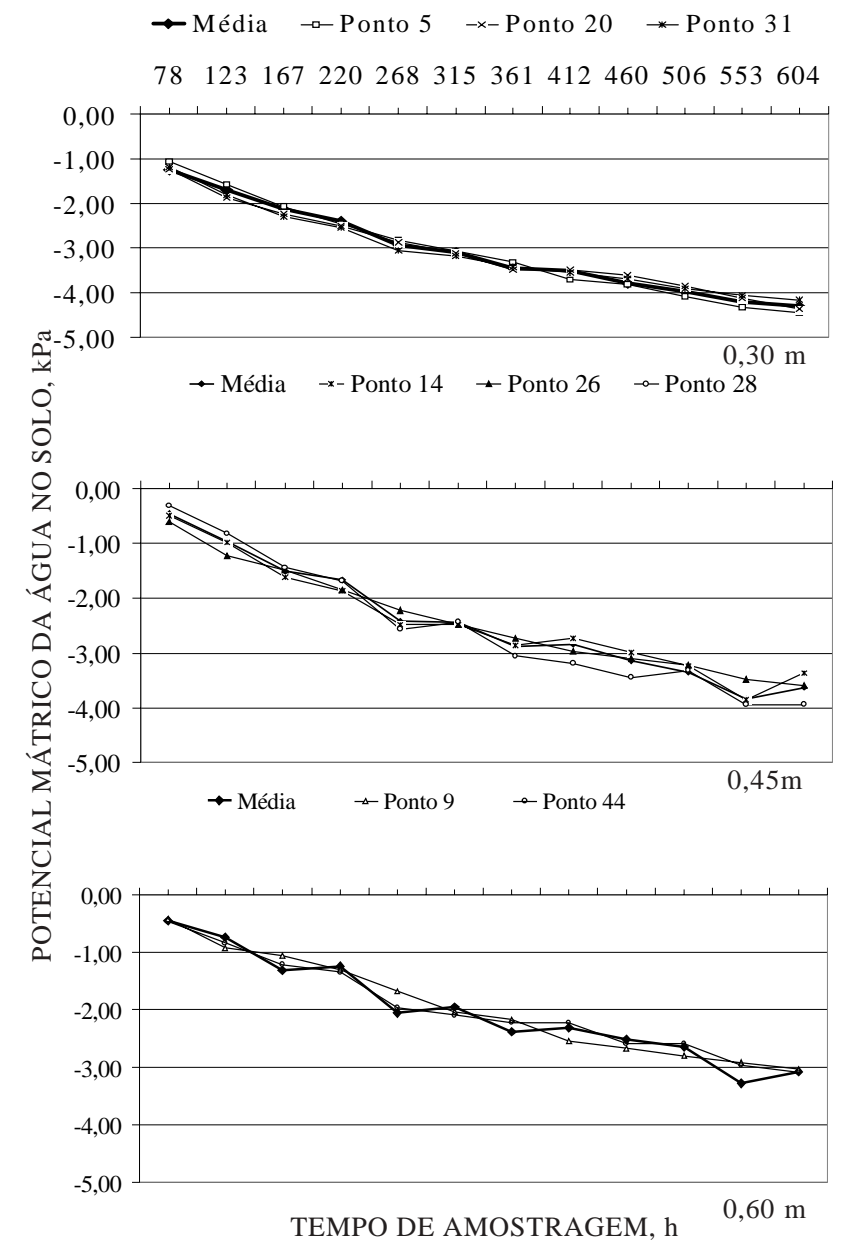

Figura 7. Comparação entre os potenciais mátricos médios da água no solo e os potenciais mátricos da água nas posições com estabilidade temporal, ao longo do tempo.

2. As posições identificadas como estáveis no tempo podem ser usadas para estimativa de $\theta$ e $\phi_{m}$ com razoável exatidão e representatividade, sendo aconsel hável a identificação de mais de um local de medida para ampliar a representatividade da amostragem.

\section{AGRADECIMENTOS}

Os autores agradecem à FAPESP, pelo auxílio concedido para a execução deste projeto de pesquisa (processo número 99/0210-0).

\section{LITERATURA CITADA}

ANDERSON, S.H. \& CASSEL, D.K. Statistical and autoregressive analysis of physical properties of Portsmouth sandy loam. Soil Sci. Soc. Am. J ., 50:1096-1104, 1986. 
CADIMA Z, A.; LIBARDI, P.L. \& REICHARDT, K. Variabilidade espacial da condutividade hidráulica em um Latossolo Vermelho-Amarelo textura média, no campo. R. Bras. Ci. Solo, 4:63-66, 1980.

CAMPOS, H. Estatística experimental não-paramétrica. 4.ed. Piracicaba, ESALQ/FEALQ, 1983. 349p.

GONÇALVES, A.C.A.; FOLEGATTI, M.A. \& SILVA, A.P. Estabilidade temporal da distribuição espacial da umidade do solo em área irrigada por pivô central. R. Bras. Ci. Solo, 23:155-164, 1999.

HENDRICKX, J.M.H. \& WIERENGA, P.J . Variability of soil water tension in a trickleirrigated Chile pepper field. I rrig. Sci., 11:23-30, 1990.

J ONG VAN LIER, Q. \& LIBARDI, P.L. Variabilidade dos parâmetros da relação entre condutividade hidráulica e umidade do solo determinada pelo método do perfil instantâneo. R. Bras. Ci. Solo, 23:1005-1014, 1999.

KACHANOSKI, R.G. \& DE J ONG, E. Scale dependence and the temporal persistence of spatial patterns of soil water storage. Water Res. Res., 24:85-91, 1988.

LIBARDI, P.L. Dinâmica da água no solo. 2.ed. Piracicaba: P.L. Libardi, 2000. 509p.

LIBARDI, P.L.; REICHARDT, K.; NIELSEN, D.R. \& BIGGAR, J.W. Simplified field methods for estimating the unsaturated hydraulic conductivity. Soil Sci. Soc. Am. J., 44:3-6, 1980.

LIBARDI, P.L.; MANFRON, P.A.; MORAES, S.O. \& TUON, R.L. Variabilidade da umidade gravimétrica de um solo hidromórfico. R. Bras. Ci. Solo, 20:1-12, 1996.
NIELSEN, D.R.; BIGGAR, J .W. \& ERH, K.T. Spatial variability of field measured soil water properties. Hilgardia, 42:215259, 1973.

SANTOS, H.L. \& VASCONCELOS, C.A. Determinação do número de amostras de solo para análise química em diferentes condições de manejo. R. Bras. Ci. Solo, 11:97100, 1987.

STATISTICAL ANALYSIS SYSTEM INSTITUTE. SAS/STAT Procedure guide for personal computers. Version 5, SAS Inst. Cary, 1991.

THOMSEN, A.; HANSEN, B. \& SCHELDE, K. Application of TDR to water level measurement. J . Hydrol., 236:252-258, 2000.

TOPP, G.C.; DAVIS, J .L. \& ANNAN, A.P. Electromagnetic determination of soil water content: Measurement in coaxial transmission lines. Water Res. Res., 16:574-582, 1980.

VACHAUD, G.; PASSERAT DE SILANS, A.; BALABANIS, P. $\&$ VAUCLIN, M. Temporal stability of spatially measured soil water probability density function. Soil Sci. Soc. Am. J ., 49:822-827, 1985.

VAN WESENBEECK, I.J .; KACHANOSKI, R.G. \& ROLSTON, D.E. Temporal persistence of spatial patterns of soil water content in the tilled layer under a corn crop. Soil Sci. Soc. Am. J ., 52:934-941, 1988.

WARRICK, A.W. \& NIELSEN, D.R. Spatial variability of soil physical properties in the field. In: HILLEL, D., ed. Applications of soil physics. New York, Academic Press, 1980. p.319-344. 